\title{
Handbook of Porphyrin Science
}

with Applications to Chemistry, Physics,

Materials Science, Engineering, Biology and Medicine 
This page intentionally left blank 


\title{
Handbook of \\ Porphyrin Science
}

with Applications to Chemistry, Physics, Materials Science, Engineering, Biology and Medicine

\author{
শ্రি \\ Volume 16 \\ Synthetic Developments \\ Part I
}

\author{
Editors \\ Karl M. Kadish \\ University of Houston, USA \\ Kevin M. Smith \\ Louisiana State University, USA \\ Roger Guilard \\ Université de Bourgogne, France
}

\section{No world Scientific}




\section{Published by}

World Scientific Publishing Co. Pte. Ltd.

5 Toh Tuck Link, Singapore 596224

USA office: 27 Warren Street, Suite 401-402, Hackensack, NJ 07601

UK office: 57 Shelton Street, Covent Garden, London WC2H 9HE

\section{British Library Cataloguing-in-Publication Data}

A catalogue record for this book is available from the British Library.

\section{HANDBOOK OF PORPHYRIN SCIENCE with Applications to Chemistry, Physics, Materials Science, Engineering, Biology and Medicine (Volumes 16-20)}

Copyright (C) 2012 by World Scientific Publishing Co. Pte. Ltd.

All rights reserved. This book, or parts thereof, may not be reproduced in any form or by any means, electronic or mechanical, including photocopying, recording or any information storage and retrieval system now known or to be invented, without written permission from the Publisher.

For photocopying of material in this volume, please pay a copying fee through the Copyright Clearance Center, Inc., 222 Rosewood Drive, Danvers, MA 01923, USA. In this case permission to photocopy is not required from the publisher.

ISBN-13 978-981-4335-49-2 (Set)

ISBN-10 981-4335-49-5 (Set)

ISBN-13 978-981-4335-51-5 (Vol. 16)

ISBN-10 981-4335-51-7 (Vol. 16)

Typeset by Stallion Press

Email: enquiries@stallionpress.com

Printed in Singapore. 


\section{Contents}

Preface for Volumes 16-20

ix

Preface for Volumes 1-10

Contributing Authors

$\mathrm{x}$

Contents of Volumes 1-20

$x i$

xxix

\section{4 / Carbaporphyrins and Related Systems. Synthesis, Characterization, Reactivity and Insights into Porphyrinoid Aromaticity}

\section{Timothy D. Lash}

List of Abbreviations. . . . . . . . . . . . . . . . . . . . . . 2

I. Introduction . . . . . . . . . . . . . . . . . . 3

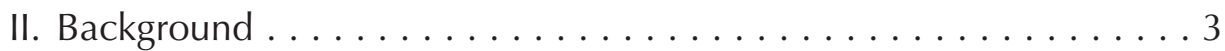

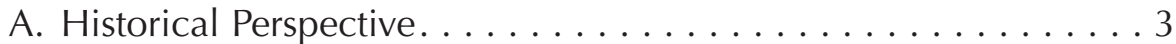

B. Nomenclature . . . . . . . . . . . . . . . . . . . . . . 8

C. General Synthetic Methods. . . . . . . . . . . . . . . . 10

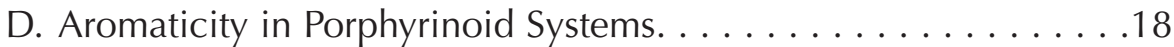

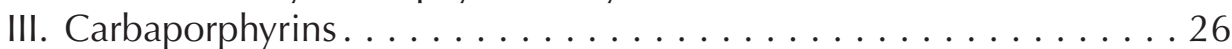

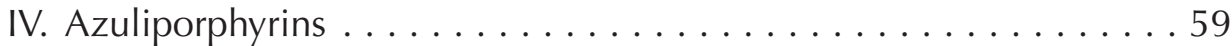

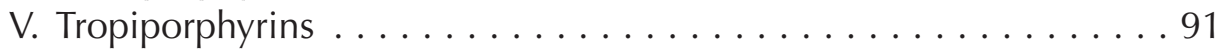

VI. Porphyrin Analogs with Six-Membered Rings . . . . . . . . . . . 98

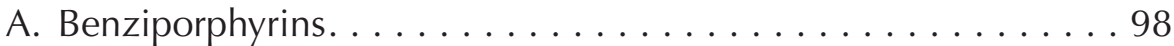

1. meso-Unsubstituted Benziporphyrins . . . . . . . . . . . 98

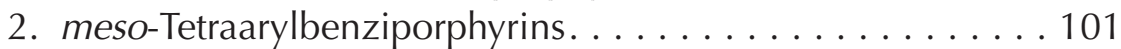

3. Dimethoxybenziporphyrins . . . . . . . . . . . . . 110

4. Oxybenziporphyrins ... . . . . . . . . . . . . . 119

5. Further Oxidized Benziporphyrins . . . . . . . . . . 133

6. 22-Hydroxybenziporphyrins. . . . . . . . . . . . . 148

7. Benziporphyrins with Exocyclic Double Bonds . . . . . . . 151

8. Benziphthalocyanines . . . . . . . . . . . . . . . 153

9. p-Benziporphyrins................... 156 
B. Naphthalene-Containing Porphyrinoid Systems . . . . . . . . . 161

1. Naphthiporphyrin . . . . . . . . . . . . . . . 161

2. Oxynaphthiporphyrins.................... . 165

3. 1,4-Naphthiporphyrins. . . . . . . . . . . . 171

C. Pyriporphyrins . . . . . . . . . . . . . . . . . . 177

VII. Carbaporphyrinoid Systems with External Heteroatoms. . . . . . . . 197

A. N-Confused Porphyrins. . . . . . . . . . . . . . . . . 197

B. O- and S-Confused Heteroporphyrins . . . . . . . . . . 221

C. Pyrazoloporphyrins . . . . . . . . . . . . . . . . 231

VIII. Neo-Confused Porphyrins . . . . . . . . . . . . . . . . . . . . . . . 241

IX. Systems with Two or More Internal Carbons . . . . . . . . . . . . 245

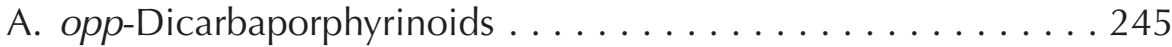

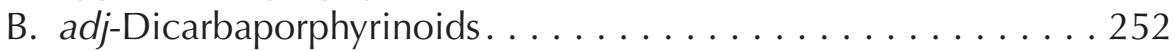

C. Toward the Synthesis of Tetracarbaporphyrinoid Systems . . . . 267

$X$. Deaza- and Dideazaporphyrins: Further Insights into the Aromatic Characteristics of Porphyrinoid Systems. . . . . . . . . 275

XI. Carbaporphyrinoids and Related Systems with Expanded or Contracted Cores . . . . . . . . . . . . . . . . . . . . . 279

A. Vinylogous Porphyrins . . . . . . . . . . . . . . . . . . 279

B. Expanded Carbaporphyrinoid Systems. . . . . . . . . . . . 284

C. Contracted Carbaporphyrinoids . . . . . . . . . . . 304

XII. Concluding Remarks. . . . . . . . . . . . . . . . . . 314

XIII. Acknowledgments . . . . . . . . . . . . . . . . . . . . 315

XIV. References . . . . . . . . . . . . . . . . . . . . . . . . 315

75 / Synthesis and Properties of the Hybrid PhthalocyanineTetrabenzoporphyrin Macrocycles Andrew N. Cammidge, Isabelle Chambrier, Michael J. Cook and Lydia Sosa-Vargas

List of Abbreviations. . . . . . . . . . . . . . . . . . . . . . . . . . . . 332

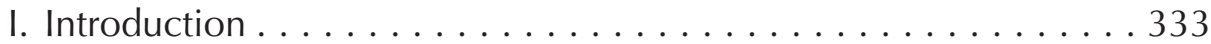

A. Nomenclature, Terminology and Notation . . . . . . . . . . . 333

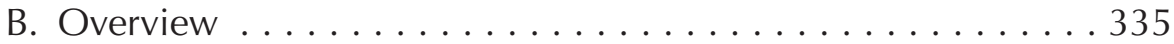

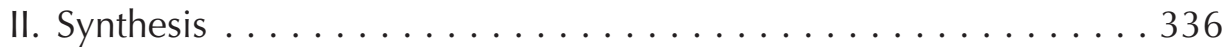

III. Molecular Properties. . . . . . . . . . . . . . . . . . . 352

A. General Solution Phase Properties and Reactivities . . . . . . . 352

B. Theoretical Treatments of Electronic Properties . . . . . . . . 357

C. UV-Vis Spectroscopy . . . . . . . . . . . . . . . . . . . 360

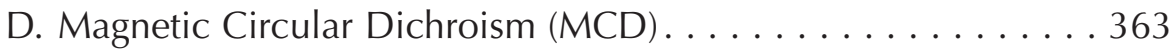


E. Photophysics . . . . . . . . . . . . . . . . . . . 365

F. Other Spectroscopies . . . . . . . . . . . . . . . . . . . 371

G. Electrochemistry and Spectroelectrochemistry . . . . . . . . 372

IV. Solid State Properties . . . . . . . . . . . . . . . . . . . . . 375

A. X-ray Structure Analyses . . . . . . . . . . . . . . . . . . . 375

B. Mesophase Properties. . . . . . . . . . . . . . . . . . . 383

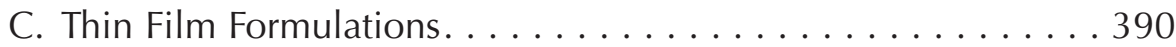

D. Electrical Measurements . . . . . . . . . . . . . . . . . 392

V. Potential Applications and Prospects . . . . . . . . . . . . 397

VI. Acknowledgments . . . . . . . . . . . . . . . . 400

VII. References . . . . . . . . . . . . . . . . . . . 400

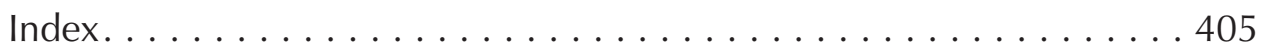


This page intentionally left blank 


\section{Preface for Volumes 16-20}

It is now more than two years and 15 volumes since we wrote our first Preface for the "Handbook of Porphyrin Science". We are delighted with the initial 15 volumes, with the diligence, timeliness, breadth of expertise, writing talent, and resolute dedication of our authors, and with the exceptional quality of the production provided by World Scientific Publishing Company. Given these successes, it was with enthusiasm that we took on the recruitment of new authors for volumes 16-20; indeed, we are extremely gratified with the responses we continue to receive to our invitations, with virtually all invitees from all over the world accepting our requests for authoritative contributions.

We are continuing with another five-volume set (Volumes 16-20) of the Handbook, with at least another five, probably ten, volumes to follow. Once again our contributors have far exceeded our expectations with regard to both quality of writing and comprehensive achievement of their individual tasks. We again asked our contributors to write "a review for the ages", and we anticipate that, as with the past 15 volumes, what you now see will remain relevant for at least 15 years. After that, maybe we will have to start all over again with a continuing compendium of the very latest and most enduring "Porphyrin Science".

The three editors once again wish to thank many individuals - our authors, our copy editors, our production team - and also World Scientific Publishing Company, for continuing to believe, with us, in the fundamental importance of porphyrins, related tetrapyrroles, and of their wide applications. We hope our readers will enjoy browsing these pages as much as we, the editors, enjoyed organizing the process that produced them.

Karl M. Kadish (Houston, USA) Kevin M. Smith (Baton Rouge, USA) Roger Guilard (Dijon, France)

February 7, 2012 


\section{Preface for Volumes 1-10}

Although the porphyrin and tetrapyrrole research area was regarded as "fully matured" during the $20^{\text {th }}$ century, as evidenced for example by the awarding of numerous Nobel Prizes to its principal researchers, new advances and accomplishments in the field still amaze us as editors. The area continues to blossom and to expand into new areas of science and applications that would probably never have occurred to our $20^{\text {th }}$ century heroes. An earlier Porphyrin Handbook assembled the large amount of factual data that had been accumulated during the $20^{\text {th }}$ century. Our new venture, the Handbook of Porphyrin Science takes a completely new look at our research area and comprehensively details the contemporary science now appearing in the scientific literature that would indeed have been hard to predict even 10 years ago. In particular, fundamentally new methodologies and potential commercial applications of the beautiful compounds that we all love are exemplified, fully recognizing the subtitle of the series - "with applications to chemistry, physics, materials science, engineering, biology and medicine".

The three of us have complementary expertise in physical chemistry, synthetic and bioorganic chemistry, and in synthetic and mechanistic organometallic chemistry; this has enabled us to cover the whole field of porphyrin science and applications, and to devise comprehensive volume and author content. As of the date of writing, between the three of us, we have published more than 1600 tetrapyrrole research articles, and hold 31 patents related to commercial applications of porphyrin science. So we do know our field, and this has enabled us to assemble a firstrate group of experts who have written comprehensive up-to-date chapters with accuracy and authority; we thank our authors for their cooperation and willingness to go along with our highly ambitious schedule for production of these volumes.

We look forward to comments from our readers, and to suggestions that might enable us to expand our basic interests and scientific coverage even further. Meanwhile, we hope that porphyrin researchers, old, new and of the future, will enjoy reading these volumes just as much as we enjoyed planning and, with the help of World Scientific Publishing Company, producing them from manuscript to published article, in a timely manner.

Karl M. Kadish (Houston, Texas, USA) Kevin M. Smith (Baton Rouge, Louisiana, USA) Roger Guilard (Dijon, Bourgogne, France) 


\section{Contributing Authors*}

\author{
Hasrat Ali \\ Université de Sherbrooke \\ Sherbrooke, Québec, Canada \\ Chapter 16

\section{Cristina Alonso} \\ University of Hull \\ Kingston-upon-Hull, HU6 7RX, UK \\ Chapter 17
}

\section{Edith Antunes}

Rhodes University

Grahamstown, 6139, South Africa

Chapter 34

\section{Naoki Aratani}

Kyoto University

Kyoto 606-8502, Japan

aratani@kuchem.kyoto-u.ac.jp

Chapter 1

\section{Margarida Archer}

Universidade Nova de Lisboa

Apartado 127

2781-901 Oeiras, Portugal

Chapter 89

\section{Katsuhiko Ariga}

National Institute for Materials Science

Namiki 1-1, Tsukuba

Ibaraki 305-0044, Japan

ARIGA.Katsuhiko@nims.go.jp

Chapter 81

\author{
Kunio Awaga \\ Nagoya University \\ Furo-cho, Chikusa-ku \\ Nagoya 464-8602, Japan \\ awaga@mbox.chem.nagoya-u.ac.jp \\ Chapter 83
}

\section{Yosra M. Badiei}

The Johns Hopkins University

Baltimore, Maryland 21218, USA

Chapter 66

\section{Teodor Silviu Balaban}

Aix-Marseille University

13397 Marseille, France

ts.balaban@univ-amu.fr

Chapter 3

\author{
Alan L. Balch \\ University of California, Davis \\ Davis, CA 95616, USA \\ balch@chem.ucdavis.edu \\ Chapter 40
}

\section{David P. Ballou}

University of Michigan

Ann Arbor, MI 48109-5606, USA

Chapter 28

\author{
Kylie D. Barker \\ University of Maryland \\ Baltimore, MD 21201-1180, USA \\ Chapter 72
}

*Full contact information for authors can be found on the title page of each chapter. 


\section{Ines Batinic'-Haberle}

Duke University Medical School Durham NC 27710, USA

ibatinic@duke.edu

Chapter 52

\section{Fethi Bedioui}

Université Paris Descartes

75231 Paris cedex 05, France

fethi-bedioui@enscp.fr

Chapter 55

\section{Ludmil Benov \\ Kuwait University \\ 13110 Safat, Kuwait \\ Chapter 52 \\ Timothy C. Berto \\ University of Michigan, \\ Ann Arbor, MI 48109, USA \\ Chapter 63}

\section{Yongzhong Bian}

University of Science and Technology Beijing

Beijing 100083, China

Chapter 64

\section{Sara Bonacchi}

Università degli Studi di Bologna

40126 Bologna, Italy

Chapter 57

\section{Véronique Bulach}

Université de Strasbourg

67000 Strasbourg, France

Chapter 62

\section{Faye Bowles}

University of California, Davis

Davis, CA 95616, USA

Chapter 40

\author{
Ross W. Boyle \\ University of Hull \\ Kingston-upon-Hull, HU6 7RX, UK \\ r.w.boyle@hull.ac.uk
}

Chapter 17

\section{Ozguncem Bozkulak}

Childrens Hospital Los Angeles

Los Angeles, CA 90027, USA

Chapter 22

\section{Martin Bröring}

Technische Universität CaroloWilhelmina zu Braunschweig

Hagenring 30, Braunschweig, Germany

m.broering@tu-bs.de

Chapter 41

\section{Markus J. Bröcker}

Yale University

266 Whitney Avenue New Haven

CT 06511, USA

Chapter 94

\section{Christian Brückner}

University of Connecticut, Storrs

CT 06369-3060, USA

c.bruckner@uconn.edu

Chapter 76 
Donald A. Bryant

The Pennsylvania State University

University Park

PA 16802, USA

dab14@psu.edu

Chapter 96

\section{Christophe Bucher}

Université Joseph Fourier/CNRS

(UMR 5250)

Grenoble, France

christophe.bucher@ujf-grenoble.fr

Chapter 78

Mihai Buda

"POLITEHNICA" University of Bucharest

Calea Grivitei 132, 010737

Bucharest, Romania

mihai@ catedra.chfiz.pub.ro

Chapter 78

\section{Kevin Burgess}

Texas A\&M University

College Station, TX 77842, USA

burgess@tamu.edu

Chapter 37

\section{Andrew N. Cammidge}

University of East Anglia

Norwich, NR4 7TJ, UK

a.cammidge@uea.ac.uk

Chapter 75

\section{José A.S. Cavaleiro}

University of Aveiro

3810-193 Aveiro, Portugal

jcavaleiro@ua.pt

Chapter 9

\section{Isabelle Chambrier}

University of East Anglia

Norwich, NR4 7TJ, UK

i.fernandes@uea.ac.uk

Chapter 75

\section{Caiyong Chen}

University of Maryland

Maryland 20742, USA

Chapter 67

\author{
Andrei V. Cheprakov \\ Moscow State University \\ 119992, Moscow, Russia \\ avchep@elorg.chem.msu.ru \\ Chapter 58
}

\section{Sung Cho}

Yonsei University

Seoul, 120-747, Korea

Chapter 5

\section{Daniel P. Collins}

University of South Carolina

Columbia, SC 29208, USA

Chapter 28

\section{Michael J. Cook \\ University of East Anglia \\ Norwich, NR4 7TJ, UK \\ M.Cook@uea.ac.uk}

Chapter 75

\section{John H. Dawson}

University of South Carolina

Columbia, SC 29208, USA

dawson@mail.chem.sc.edu

Chapter 28 


\section{Simon Dalgleish}

Nagoya University, Furo-cho

Chikusa-ku, Nagoya 464-8602, Japan

Chapter 83

\section{Serena DeBeer}

Cornell University

Ithaca, NY 14853, USA

Chapter 66

\section{Ilia G. Denisov}

The University of Illinois

Urbana, IL 61801, USA

Denisov@illinois.edu

Chapter 26

\section{Charles Michael Drain}

Hunter College of The City University of New York

New York, NY 10065, USA

cdrain@hunter.cuny.edu

Chapter 15

\section{Francis D'Souza}

University of North Texas Denton, TX 76203-5017, USA

Francis.DSouza@UNT.edu

Chapter 4

\section{Florence Duclairoir}

Institut Nanosciences et Cryogénie 38054 Grenoble cedex 9, France

Chapter 47

\section{Alessandro D'urso}

Università di Catania

95125 Catania, Italy

Chapter 59

\section{Jennifer L. DuBois}

University of Notre Dame

Notre Dame, Indiana 46556, USA

jdubois@nd.edu

Chapter 90

\section{Johannes A. A. W. Elemans}

Radboud University Nijmegen

Heyendaalseweg 135

6525 AJ Nijmegen

The Netherlands

Chapter 79

\section{Manivannan Ethirajan}

Roswell Park Cancer Institute

Buffalo, NY 14263, USA

Chapter 19

\section{Alessandro Feis \\ University of Florence \\ I-50019 Sesto Fiorentino, Italy \\ Chapter 31}

\section{Angela Ferrario}

Childrens Hospital Los Angeles

Los Angeles, CA 90027, USA

Chapter 22

\section{Gloria C. Ferreira}

University of South Florida

Tampa, Florida, 33612, USA

gferreir@health.usf.edu

Chapter 68

\section{Stuart J. Ferguson}

University of Oxford

South Parks Road

Oxford, OX1 3QU, UK

stuart.ferguson@bioch.ox.ac.uk

Chapter 93 
Kimberly B. Fields

University of South Florida

Tampa, FL 33620, USA

Chapters 13, 43

\section{Takamitsu Fukuda}

Osaka University

Toyonaka 560-0043, Japan

tfukuda@chem.sci.osaka-u.ac.jp

Chapter 42

\author{
Shunichi Fukuzumi \\ Osaka University \\ Suita, Osaka 565-0871, Japan \\ fukuzumi@chem.eng.osaka-u.ac.jp \\ Chapter 46
}

\section{Hiroyuki Furuta}

Kyushu University

Fukuoka 819-0395, Japan

hfuruta@cstf.kyushu-u.ac.jp

Chapter 10

\section{Mary Grace I. Galinato}

University of Michigan,

Ann Arbor, MI 48109, USA

Chapter 63

\section{Damiano Genovese}

Università degli Studi di Bologna

40126 Bologna, Italy

Chapter 57

\section{Jean-Paul Gisselbrecht}

Université de Strasbourg

67000 Strasbourg, France

gissel@unistra.fr

Chapter 14

\section{David P. Goldberg}

The Johns Hopkins University

Baltimore, Maryland 21218, USA

dpg@jhu.edu

Chapter 66

\section{Charles J. Gomer}

University of Southern California

Los Angeles, CA 90027, USA

Chapter 22

\section{Lauren E. Goodrich}

University of Michigan

Ann Arbor, MI 48109, USA

Chapter 63

\section{Bruno Grimm}

Friedrich-Alexander-University Erlangen-Nuremberg

91058 Erlangen, Germany

Chapter 2

\section{Sophie Griveau}

Université Paris Descartes

75231 Paris cedex 05, France

Chapter 55

\section{Roger Guilard}

Université de Bourgogne

21078 Dijon Cedex, France

Roger.Guilard@u-bourgogne.fr

Chapter 49

\section{Dirk M. Guldi}

Friedrich-Alexander-University

Erlangen-Nuremberg

91058 Erlangen, Germany

dirk.guldi@chemie.uni-erlangen.de

Chapter 2 


\section{Victoria L. Gunderson}

Northwestern University

Evanston IL 60208-3113, USA

Chapter 95

\section{Iqbal Hamza}

University of Maryland

Maryland 20742, USA

hamza@umd.edu

Chapter 67

\section{Pierre D. Harvey}

Université de Sherbrooke

Sherbrooke, J1K 2R1, Québec, Canada

Chapter 49

\section{Anita Hausmann}

Friedrich-Alexander-University

Erlangen-Nuremberg

91058 Erlangen, Germany

Chapter 2

\section{Takashi Hayashi}

Osaka University

Suita 565-0871, Japan

thayashi@chem.eng.osaka-u.ac.jp

Chapter 23

\section{Petra Hellwig}

Université de Strasbourg 67000 Strasbourg, France

hellwig@chimie.u-strasbg.fr

Chapter 36

\section{Jonathan P. Hill}

National Institute for Materials Science

Namiki 1-1, Tsukuba

Ibaraki 305-0044, Japan

Jonathan.HILL@nims.go.jp

Chapter 81

\section{Yoshio Hisaeda}

Kyushu University

Fukuoka 819-0395, Japan

yhisatcm@mail.cstm.kyushu-u.ac.jp

Chapter 48

\section{Seong-Jin Hong}

Kangwon National University

Chun-Chon, 200-701, Korea

Chapter 60

\section{Mir Wais Hosseini}

Université de Strasbourg

67000 Strasbourg, France

hosseini@chimie.u-strasbg.fr

Chapter 62

\section{Barry D. Howes}

University of Florence

I-50019 Sesto Fiorentino, Italy

Chapter 31

\section{Gregory A. Hunter}

University of South Florida

Tampa, Florida, 33612, USA

Chapter 68

\section{Jotaro Igarashi}

Tohoku University

Katahira, Sendai 980-8577, Japan

Chapter 73
Akira Ikezaki
Toho University
Ota-ku, Tokyo 143-8540, Japan
Chapter 32 


\section{Shinsuke Ishihara}

National Institute for Materials Science

Namiki 1-1, Tsukuba

Ibaraki 305-0044, Japan

Chapter 81

\section{Osamu Ito}

Tohoku University

Sendai, 981-3215, Japan

Chapter 4

Hisashi Ito

Hokkaido University

N19 W8, Kita-ku

Sapporo 060-0819

Japan

Chapter 98

\section{Anabella Ivancich}

Centre Nationale de la Recherche Scientifique

F-91191 Gif-sur-Yvette, France

Chapter 31

\section{Hiroshi Imahori}

Kyoto University, Nishikyo-ku

Kyoto 615-8510, Japan

imahori@scl.kyoto-u.ac.jp

Chapter 80

\section{Dieter Jahn}

Technische Universität Braunschweig

Spielmannstrasse 7

D-38106 Braunschweig

Germany

d.jahn@tu-bs.de

Chapters 70, 94

\section{Martina Jahn}

Technische Universität Braunschweig

D-38106 Braunschweig, Germany

Chapter 70

\section{Christophe Jeandon}

Université de Strasbourg

67000 Strasbourg, France

cjeandon@unistra.fr

Chapter 14

\section{Jianzhuang Jiang}

University of Science and Technology Beijing

Beijing 100083, China

jianzhuang@ustb.edu.cn

Chapter 64

\section{Riccardo Juris}

Università degli Studi di Bologna

40126 Bologna, Italy

Chapter 57

\section{Huangxian Ju}

Nanjing University

Nanjing 210093, China

hxju@nju.edu.cn

Chapter 82

\section{Norbert Jux}

Universität Erlangen-Nürnberg

91054 Erlangen, Germany

norbert.jux@chemie.uni-erlanger.de

Chapter 20

\section{Axel Kahnt}

Friedrich-Alexander-University Erlangen-Nuremberg

91058 Erlangen, Germany

Chapter 2 


\section{Toshiaki Kamachi}

Tokyo Institute of Technology

Yokohama 226-8501, Japan

Chapter 56

\section{Yoriko Kanai}

Shinshu University

3-15-1 Tokida, Ueda, 386-8567, Japan

Chapter 53

\section{David Kessel}

Wayne State University School of Medicine

Detroit, MI 48201, USA

dhkessel@med.wayne.edu

Chapter 21

\section{Dongho Kim}

Yonsei University

Seoul, 120-747, Korea

dongho@yonsei.ac.kr

Chapters 5, 6

\section{Kil Suk Kim}

Yonsei University

Seoul, 120-747, Korea

Chapter 6

\section{Kenichi Kitanishi}

Tohoku University

Katahira, Sendai 980-8577, Japan

Chapter 73

\section{Nagao Kobayashi}

Tohoku University

Sendai 980-8578, Japan

nagaok@mail.tains.tohoku.ac.jp

Chapters 33, 42

\section{Koichi Kobayashi}

Plant Science Center

RIKEN Institute, Japan

kouity@pcs.riken.jp

Chapter 97

\section{Kei Kurotobi}

Kyoto University, Nishikyo-ku

Kyoto 615-8510, Japan

Chapter 80

\section{Michio Kunieda}

Ritsumeikan University

Kusatsu, Shiga 525-8577, Japan

Chapter 51

\section{Timothy D. Lash}

Illinois State University

Normal, Illinois 61790-4160, USA

tdlash@ilstu.edu

Chapter 74

\section{Lechosław Latos-Grażyński}

University of Wrocław

Wrocław 50 383, Poland

1lg@wchuwr.pl

Chapter 8

\section{Janet T. F. Lau}

The Chinese University of Hong Kong Shatin, N.T., Hong Kong, China

Chapter 84

\section{Rosaria Lauceri}

Università di Catania

95125 Catania, Italy

Chapter 59 


\section{Gunhild Layer}

Technische Universität Braunschweig

D-38106 Braunschweig, Germany

g.layer@tu-braunschweig.de

Chapters 70, 88

\section{Chang-Hee Lee}

Kangwon National University

Chun-Chon, 200-701, Korea

Chapter 60

\section{Pannee Leeladee}

The Johns Hopkins University

Baltimore, Maryland 21218, USA

Chapter 66

\section{Nicolai Lehnert}

University of Michigan,

Ann Arbor, MI 48109, USA

lehnert@umich.edu

Chapter 63

\section{Jianping Lei}

Nanjing University

Nanjing 210093, China

jpl@nju.edu.cn

Chapter 82

\section{Genxi Li}

Nanjing University

Nanjing 210093, PR China

genxili@nju.edu.cn

Chapter 27

\section{Jong Min Lim}

Yonsei University

Seoul, 120-747, Korea

Chapter 6

\section{Zhenfeng Liu}

The Pennsylvania State University

University Park

PA 16802, USA

Chapter 96

\section{Pui-Chi Lo}

The Chinese University of Hong Kong

Shatin, N.T., Hong Kong, China

pclo@cuhk.edu.hk

Chapter 84

\section{Susana A. Lobo}

Universidade Nova de Lisboa

Apartado 127

2781-901 Oeiras, Portugal

Chapter 89

\section{Aurore Loudet}

Texas A\&M University

College Station, TX 77842, USA

Chapter 37

\section{Ricardo O. Louro}

Universidade Nova de Lisboa

Apartado 127

2781-901 Oeiras, Portugal

Chapter 89

\section{Evgeny A. Lukyanets}

Organic Intermediates and Dyes Institute Moscow, 123995, Russia rmeluk@niopik.ru

Chapter 11

\section{Marian Luna}

Childrens Hospital Los Angeles

Los Angeles, CA 90027, USA

Chapter 22 
Stuart A. MacGowan

Trinity College Dublin

Dublin 2, Ireland

Chapter 61

\section{Hiromitsu Maeda}

Ritsumeikan University

Kusatsu 525-8577, Japan

maedahir@ph.ritsumei.ac.jp

Chapter 38

\section{Fabrizio Mancin}

Università degli Studi di Bologna 40126 Bologna, Italy

Chapter 57

\section{Jean-Claude Marchon}

Institut Nanosciences et Cryogénie 38054 Grenoble cedex 9, France jean-claude.marchon@cea.fr

Chapter 47

\section{Michael A. Marletta}

University of California, Berkeley

Berkeley, USA

marletta@berkeley.edu

Chapter 69

\section{Victoria Martínez-Díaz}

Universidad Autónoma de Madrid 28049-Madrid, Spain

Chapter 45

\section{Tatsuru Masuda}

The University of Tokyo

Meguro, Tokyo 153-8902, Japan

ctmasuda@mail.ecc.u-tokyo.ac.jp

Chapter 97

\section{Pedro M. Matias}

Universidade Nova de Lisboa

Apartado 127

2781-901 Oeiras, Portugal

matias@itqb.unl.pt

Chapter 89

\section{A. Grant Mauk}

University of British Columbia, Vancouver

British Columbia V6T 1 Z3 Canada

mauk@exchange.ubc.ca

Chapter 92

\section{Jeffery A. Mayfield}

University of Notre Dame

Notre Dame, Indiana 46556, USA

Chapter 90

\section{Amanda J. McGown}

The Johns Hopkins University

Baltimore, Maryland 21218, USA

Chapter 66

\section{Craig J. Medforth}

University of New Mexico

Albuquerque, NM 87106, USA

Chapter 50

\section{Takashi Megumi}

Shinshu University

3-15-1 Tokida, Ueda, 386-8567, Japan

Chapter 53

\section{Frederic Melin}

Université de Strasbourg

67000 Strasbourg, France

Chapter 36 


\section{Andrey Mironov}

Lomonosov Moscow State University of Fine Chemical Technologies

119571, 86, Vernadsky’s Avenue

Moscow, Russia

mironov@mitht.ru

Chapter 85

\section{Marco Montalti}

Università degli Studi di Bologna

40126 Bologna, Italy

Chapter 57

\section{Donato Monti}

University of Rome "Tor Vergata"

00133 Rome, Italy

Chapter 54

\section{Jürgen Moser}

Technische Universität Braunschweig

Spielmannstrasse 7

D-38106 Braunschweig

Germany

j.moser@tu-bs.de

Chapter 94

\section{Shingo Nagano}

Tottori University

Tottori 680-8552, Japan

Chapter 25

\section{Mikio Nakamura}

Toho University

Ota-ku, Tokyo 143-8540, Japan

mnakamu@med.toho-u.ac.jp

Chapter 32

\section{Wonwoo Nam}

Ewha Womans University

Seoul 120-750, South Korea

wwnam@ewha.ac.kr

Chapter 44

\section{Sara Nardis}

University of Rome "Tor Vergata"

00133 Rome, Italy

Chapter 54

\section{Corrado Di Natale}

University of Rome "Tor Vergata"

00133 Rome, Italy

Chapter 54

\section{Victor N. Nemykin}

University of Minnesota Duluth

Duluth, MN 55812, USA

vnemykin@d.umn.edu

Chapter 11

\section{Maria G.P.M.S. Neves}

University of Aveiro

3810-193 Aveiro, Portugal

Chapter 9

\section{Huy-Danh Nguyen-Tran}

Shinshu University

3-15-1 Tokida, Ueda, 386-8567, Japan

Chapter 53

\section{Dennis K. P. Ng}

The Chinese University of Hong Kong Shatin, N.T., Hong Kong, China

dkpn@cuhk.edu.hk

Chapter 84 


\section{Roeland J. M. Nolte}

Radboud University Nijmegen

Heyendaalseweg 135

6525 AJ Nijmegen

The Netherlands

r.nolte@science.ru.nl

Chapter 79

\section{Tebello Nyokong}

Rhodes University

Grahamstown, 6139, South Africa

t.nyokong@ru.ac.za

Chapter 34

\section{Junichi Ogikubo}

University of Connecticut, Storrs

CT 06369-3060, USA

Chapter 76

\section{Yoshiki Ohgo}

Toho University

Ota-ku, Tokyo 143-8540, Japan

Chapter 32

\section{Kazuchika Ohta}

Shinshu University

3-15-1 Tokida, Ueda, 386-8567, Japan

ko52517@shinshu-u-ac.jp

Chapter 53

\section{Tetsuo Okujima}

Ehime University

Matsuyama 790-8577, Japan

Chapter 7

\section{Ichiro Okura}

Tokyo Institute of Technology

Yokohama 226-8501, Japan

iokura@bio.titech.ac.jp

Chapter 56

\section{Noboru Ono}

Ehime University

Matsuyama 790-8577, Japan

ononbr@dpc.ehime-u.ac.jp

Chapter 7

\author{
Atsuhiro Osuka \\ Kyoto University \\ Kyoto 606-8502, Japan \\ osuka@kuchem.kyoto-u.ac.jp \\ Chapter 1
}

\section{Zhongping Ou}

Jiangsu University

Zhenjiang 212013, China

Chapter 64

\section{Ravindra K. Pandey \\ Roswell Park Cancer Institute \\ Buffalo, NY 14263, USA \\ ravindra.pandey@ roswellpark.org \\ Chapter 19}

\section{Roberto Paolesse}

University of Rome "Tor Vergata" 00133 Rome, Italy roberto.paolesse@uniroma2.it

Chapter 54

\section{Nayan J. Patel}

Roswell Park Cancer Institute

Buffalo, NY 14263, USA

Chapter 19

\section{Miłosz Pawlicki}

University of Wrocław

Wrockaw 50 383, Poland

Chapter 8 
Inês A. C. Pereira

Universidade Nova de Lisboa

Apartado 127

2781-901 Oeiras, Portugal

Chapter 89

\section{John D. Phillips}

University of Utah School of Medicine

Salt Lake City, Utah 84132, USA

john.phillips@hsc.utah.edu

Chapter 91

\section{Thomas L. Poulos}

University of California Irvine

Irvine, California 92697-3900, USA

poulos@uci.edu

Chapter 87

\section{Luca Prodi}

Università degli Studi di Bologna

40126 Bologna, Italy

luca.prodi@unibo.it

Chapter 57

\section{Katharine A. Prokop}

The Johns Hopkins University

Baltimore, Maryland 21218, USA

Chapter 66

\section{Roberto Purrello}

Università di Catania

95125 Catania, Italy

r.purrello@unict.it

Chapter 59

\section{Stephen W. Ragsdale}

University of Michigan

Ann Arbor, MI 48109-0606, USA

sragsdal@umich.edu

Chapter 86

\section{Enrico Rampazzo}

Università degli Studi di Bologna 40126 Bologna, Italy

Chapter 57

\section{Júlio S. Rebouças}

Universidade Federal da Paraíba

João Pessoa, PB 58051-970, Brazil

Chapter 52

\section{Sébastien Richeter}

Université Montpellier 2

34095 Montpellier Cedex 5, France sebastien.richeter@univ-montp2.fr

Chapter 14

\section{Beate Röder}

Humboldt-Universität zu Berlin 12489 Berlin, Germany

roeder@physik.hu-berlin.de

Chapter 20

\section{Vladimir Roznyatovskiy}

The University of Texas at Austin

Austin, Texas, 78712, USA

Chapter 60

\section{Célia V. Romão}

Universidade Nova de Lisboa

Apartado 127

2781-901 Oeiras, Portugal

Chapter 89

Alan E. Rowan

Radboud University Nijmegen

Heyendaalseweg 135

6525 AJ Nijmegen

The Netherlands

A.Rowan@ @science.ru.nl

Chapter 79 


\section{Natalie Rucker}

Childrens Hospital Los Angeles

Los Angeles, CA 90027, USA

Chapter 22

\section{Alexander B. Rudine}

Portland State University, Portland

OR 97207-0751, USA

Chapter 80

\section{Joshua V. Ruppel}

University of South Florida

Tampa, FL 33620, USA

Chapters 13, 43

\section{Romain Ruppert}

Université de Strasbourg

67000 Strasbourg, France

rruppert@unistra.fr

Chapter 14

\section{Aoife Ryan}

Trinity College Dublin

Dublin 2, Ireland

Chapter 12

\section{Lalith Samankumara}

University of Connecticut, Storrs

CT 06369-3060, USA

Chapter 76

\section{Lígia M. Saraiva}

Universidade Nova de Lisboa

Apartado 127

2781-901 Oeiras, Portugal

Chapter 89

\section{Wolfgang Seitz}

Friedrich-Alexander-University

Erlangen-Nuremberg

91058 Erlangen, Germany

Chapter 2

\section{Mathias O. Senge}

Trinity College Dublin

Dublin 2, Ireland

sengem@tcd.ie

Chapters 12, 61

\section{Natalia N. Sergeeva}

Trinity College Dublin

Dublin 2, Ireland

Chapter 12

\section{Jonathan L. Sessler}

The University of Texas at Austin

Austin, Texas, 78712, USA

sessler@mail.utexas.edu

Chapter 60

\section{Scott Severance}

University of Maryland

Maryland 20742, USA

Chapter 67

\section{John A. Shelnutt}

Sandia National Laboratories

Albuquerque

NM 87185-1349, USA

jasheln@unm.edu

Chapter 50

\section{Hisashi Shimakoshi}

Kyushu University

Fukuoka 819-0395, Japan

Chapter 48

\section{Toru Shimizu}

Tohoku University

Katahira, Sendai 980-8577, Japan

shimizu@tagen.tohoku.ac.jp

Chapter 73 


\section{Jae-Yoon Shin}

Yonsei University

Seoul, 120-747, Korea

Chapter 6

\section{Yoshitsugu Shiro}

Harima Institute

Hyogo 679-5148, Japan

yshiro@riken.jp

Chapters 24, 25

\section{Martha Sibrian-Vazquez}

Portland State University

Portland, OR 97201, USA

Chapter 18

\section{Sunaina Singh}

Hunter College of The City University of New York

New York, NY 10065, USA

Chapter 15

\section{Dariusz A. Sliwa}

University of Michigan

Ann Arbor, MI 48109-0606, USA

Chapter 86

\section{Stephen G. Sligar}

The University of Illinois

Urbana, IL 61801, USA

s-sligar@uiuc.edu

Chapter 26

\section{Ann Smith}

University of Missouri

Kansas City, MO 64110, USA

SmithAn@umkc.edu

Chapter 71

\section{Giulietta Smulevich}

University of Florence

I-50019 Sesto Fiorentino, Italy

giulietta.smulevich@unifi.it

Chapter 31

\section{Julie M. Stevens}

University of Oxford

South Parks Road

Oxford, OX1 3QU, UK

julie.stevens@bioch.ox.ac.uk

Chapter 93

\section{Nicole L. Snyder}

Hamilton College

Clinton, NY 13323, USA

Chapters 13, 43

\section{Lydia Sosa-Vargas}

University of East Anglia

Norwich, NR4 7TJ, UK

Chapter 75

\section{Fabian Spänig}

Friedrich-Alexander-University

Erlangen-Nuremberg

91058 Erlangen, Germany

Chapter 2

\section{Ivan Spasojević}

Duke University Medical School Durham

NC 27710, USA

Chapter 52

\section{Tatyana Spolitak}

University of Michigan

Ann Arbor, MI 48109-5606, USA

Chapter 28 


\section{Christine Stern}

Université de Bourgogne

21078 Dijon Cedex, France

Chapter 49

\section{Martin J. Stillman}

The University of Western Ontario

London, Ontario N6A 5B7, Canada

stillman@uwo.ca

Chapter 65

\section{Hiroshi Sugimoto}

Harima Institute

Hyogo 679-5148, Japan

Chapter 24

\section{Yasufumi Takagi}

Shinshu University

3-15-1 Tokida, Ueda, 386-8567, Japan

Chapter 53

\section{Atsushi Takabayashi}

Hokkaido University

N19 W8, Kita-ku

Sapporo 060-0819

Japan

Chapter 98

\section{Shin-ichi J. Takayama}

University of British Columbia

Vancouver

British Columbia V6T 1 Z3 Canada

Chapter 92

\section{Osamu Takikawa}

National Center for Geriatrics and Gerontology

Obu, Aichi 474-8522, Japan

Chapter 24

\section{Hitoshi Tamiaki}

Ritsumeikan University

Kusatsu, Shiga 525-8577, Japan

tamiaki@ph.ritsumei.ac.jp

Chapter 51

\author{
Ayumi Tanaka \\ Hokkaido University \\ N19 W8, Kita-ku \\ Sapporo 060-0819 \\ Japan \\ ayumi@pop.lowtem.hokudai.ac.jp \\ Chapter 98
}

\section{Ryouichi Tanaka}

Hokkaido University

N19 W8, Kita-ku

Sapporo 060-0819

Japan

rtanaka@lowtem.hokudai.ac.jp

Chapter 98

\section{Lisa Tauchi}

Shinshu University

3-15-1 Tokida, Ueda, 386-8567, Japan

Chapter 53

\section{Miguel Teixeira}

Universidade Nova de Lisboa

Apartado 127, 2781-901 Oeiras, Portugal

Chapter 89
Alison Thompson
Dalhousie University
Halifax, Nova Scotia, Canada
alison.thompson@dal.ca
Chapter 39 


\section{Motoki Toganoh}

Kyushu University

Fukuoka 819-0395, Japan

Chapter 10

\section{Augusto C. Tomé}

University of Aveiro

3810-193 Aveiro, Portugal

Chapter 9

\section{Tomas Torres}

Universidad Autónoma de Madrid 28049-Madrid, Spain tomas.torres@uam.es

Chapter 45

\section{Paola Turano}

University of Florence

I-50019 Sesto Fiorentino, Italy

turano@cerm.unifi.it

Chapter 30

\section{Md. Imam Uddin}

Dalhousie University

Halifax, Nova Scotia, Canada

Chapter 39

\section{Johan E. van Lier}

Université de Sherbrooke

Sherbrooke, Québec, Canada

Johannes.Van.Lier@USherbrooke.ca

Chapter 16

\section{Nico Veling}

Radboud University Nijmegen

Heyendaalseweg 135

6525 AJ Nijmegen

The Netherlands

Chapter 79

\section{Maria da Graça H. Vicente}

Louisiana State University

Baton Rouge, LA 70803, USA

vicente@1su.edu

Chapter 18

\section{Sam P. de Visser}

The University of Manchester

Manchester M1 7DN, UK

sam.devisser@manchester.ac.uk

Chapter 44

\section{F. Ann Walker}

University of Arizona

Tucson, AZ 85721-0041, USA

awalker@email.arizona.edu

Chapter 29

\section{Michael G. Walter}

University of North Carolina at Charlotte Charlotte, NC 28223, USA

Chapter 80

\section{Jacek Waluk}

Polish Academy of Sciences

01-224 Warsaw, Poland

waluk@ichf.edu.pl

Chapter 35

\section{Carl C. Wamser}

Portland State University, Portland

OR 97207-0751, USA

wamserc@pdx.edu

Chapter 80

\section{Martin J. Warren}

University of Kent

Canterbury, Kent, UK

m.j.warren@kent.ac.uk

Chapter 88 


\section{Michael R. Wasielewski}

Northwestern University

Evanston IL 60208-3113, USA

m-wasielewski@northwestern.edu

Chapter 95

\section{Emily E. Weinert}

University of California, Berkeley

Berkeley, USA

Chapter 69

\section{Angela Wilks}

University of Maryland

Baltimore, MD 21201-1180, USA

awilks@ rx.umaryland.edu

Chapter 72

Sam Wong

Childrens Hospital Los Angeles

Los Angeles, CA 90027, USA

Chapter 22

Tabitha E. Wood

Dalhousie University

Halifax, Nova Scotia, Canada

Chapter 39

\section{Frank Xu}

Childrens Hospital Los Angeles

Los Angeles, CA 90027, USA

Chapter 22

\section{Hiroko Yamada}

Ehime University

Matsuyama 790-8577, Japan

Chapter 7

\section{Jaesung Yang}

Yonsei University

Seoul, 120-747, Korea

Chapter 5

\section{Hyejin Yoo}

Yonsei University

Seoul, 120-747, Korea

Chapter 5

\section{Min-Chul Yoon}

Yonsei University

Seoul, 120-747, Korea

Chapter 6

\section{Zin Seok Yoon}

Yonsei University

Seoul, 120-747, Korea

Chapter 5

\section{Nelsi Zaccheroni}

Università degli Studi di Bologna 40126 Bologna, Italy

Chapter 57

\section{Peter Zhang}

University of South Florida

Tampa, FL 33620, USA

pzhang@cas.usf.edu

Chapters 13, 43

\section{Yuexing Zhang}

University of Science and Technology Beijing

Beijing 100083, China

Chapter 64

\section{Yuzhen Zhou}

University of Michigan

Ann Arbor, MI 48109-0606, USA

Chapter 86

\section{Christopher J. Ziegler}

University of Akron

Akron OH 44325-3501 USA

ziegler@uakron.edu

Chapter 77 


\section{Contents of Volumes 1-20}

\section{Volume 1 Supramolecular Chemistry}

1. Synthetic Strategies Toward Multiporphyrinic Architectures Naoki Aratani and Atsuhiro Osuka

2. Charge Transfer Between Porphyrins/Phthalocyanines and Carbon Nanostructures

Bruno Grimm, Anita Hausmann, Axel Kahnt, Wolfgang Seitz, Fabian Spänig and Dirk M. Guldi

3. Self-Assembling Porphyrins and Chlorins as Synthetic Mimics of the Chlorosomal Bacteriochlorophylls Teodor Silviu Balaban

4. Tetrapyrrole-Nanocarbon Hybrids: Self-Assembly and Photoinduced Electron Transfer Francis D'Souza and Osamu Ito

5. Photophysical Properties of Various Directly Linked Porphyrin Arrays Zin Seok Yoon, Jaesung Yang, Hyejin Yoo, Sung Cho and Dongho Kim

6. Photophysics and Photochemistry of Various Expanded Porphyrins Jong Min Lim, Min-Chul Yoon, Kil Suk Kim, Jae-Yoon Shin and Dongho Kim

\section{Volume 2 Synthesis and Coordination Chemistry}

7. Synthesis of Porphyrins Fused with Aromatic Rings Noboru Ono, Hiroko Yamada and Tetsuo Okujima

8. Carbaporphyrinoids - Synthesis and Coordination Properties Miłosz Pawlicki and Lechosław Latos-Grażyński

9. meso-Tetraarylporphyrin Derivatives: New Synthetic Methodologies José A.S. Cavaleiro, Augusto C. Tomé and Maria G.P.M.S. Neves

10. Synthesis and Metal Coordination of N-Confused and N-Fused Porphyrinoids

Motoki Toganoh and Hiroyuki Furuta 


\section{Volume 3 Synthetic Methodology}

11. The Key Role of Peripheral Substituents in the Chemistry of Phthalocyanines

Victor N. Nemykin and Evgeny A. Lukyanets

12. Organometallic $\mathrm{C}-\mathrm{C}$ Coupling Reactions for Porphyrins Natalia N. Sergeeva, Mathias O. Senge and Aoife Ryan

13. Porphyrin Functionalization via Palladium-Catalyzed Carbon-Heteroatom Cross-Coupling Reactions

Kimberly B. Fields, Joshua V. Ruppel, Nicole L. Snyder and $X$. Peter Zhang

14. Peripherally Metalated Porphyrin Derivatives: Synthetic Approaches and Properties

Sébastien Richeter, Christophe Jeandon, Jean-Paul Gisselbrecht and Romain Ruppert

15. Combinatorial Libraries of Porphyrins: Chemistry and Applications Charles Michael Drain and Sunaina Singh

\section{Volume 4 Phototherapy, Radioimmunotherapy and Imaging}

16. Porphyrins and Phthalocyanines as Photosensitizers and Radiosensitizers Hasrat Ali and Johan E. van Lier

17. Bioconjugates of Porphyrins and Related Molecules for Photodynamic Therapy Cristina Alonso and Ross W. Boyle

18. Syntheses of Boronated Porphyrins and Their Application in BNCT Maria da Graça H. Vicente and Martha Sibrian-Vazquez

19. Porphyrin-Based Multifunctional Agents for Tumor-Imaging and Photodynamic Therapy (PDT)

Manivannan Ethirajan, Nayan J. Patel and Ravindra K. Pandey

20. Targeting Strategies for Tetrapyrrole-Based Photodynamic Therapy of Tumors Norbert Jux and Beate Röder

21. Mechanisms of Cell Death in Photodynamic Therapy David Kessel

22. Photodynamic Therapy and the Tumor Microenvironment Charles J. Gomer, Angela Ferrario, Marian Luna, Natalie Rucker, Sam Wong, Ozguncem Bozkulak and Frank Xu 


\section{Volume 5 Heme Proteins}

23. Hemoproteins Reconstituted with Artificially Created Hemes Takashi Hayashi

24. Tryptophan Catabolism by Heme Dioxygenases Hiroshi Sugimoto, Osamu Takikawa and Yoshitsugu Shiro

25. NO Chemistry by Heme-Enzymes Yoshitsugu Shiro and Shingo Nagano

26. Cytochrome P450 Enzymes Ilia G. Denisov and Stephen G. Sligar

27. Heme Protein-Based Electrochemical Biosensors Genxi Li

28. The Generation and Characterization of the Compounds I and ES States of Cytochrome P450 Using Rapid Mixing Methods Daniel P. Collins, Tatyana Spolitak, David P. Ballou and John H. Dawson

\section{Volume 6 NMR and EPR Techniques}

29. NMR and EPR Spectroscopy of Paramagnetic Metalloporphyrins and Heme Proteins

F. Ann Walker

30. Heme Acquisition by Hemophores: A Lesson from NMR Paola Turano

31. Structure-Function Relationships Among Heme Peroxidases: New Insights from Electronic Absorption, Resonance Raman and Multifrequency Electron Paramagnetic Resonance Spectroscopies Giulietta Smulevich, Alessandro Feis, Barry D. Howes and Anabella Ivancich

\section{Volume 7 Physicochemical Characterization}

32. Electronic and Magnetic Structures of Iron Porphyrin Complexes Mikio Nakamura, Yoshiki Ohgo and Akira Ikezaki

33. Optically Active Porphyrin Systems Analyzed by Circular Dichroism Nagao Kobayashi

34. Photochemical and Photophysical Properties of Metallophthalocyanines Tebello Nyokong and Edith Antunes

35. Structure, Spectroscopy, Photophysics, and Tautomerism of Free-Base Porphycenes and Other Porphyrin Isomers Jacek Waluk 
36. Recent Applications of Infrared Spectroscopy and Microscopy in Chemistry, Biology and Medicine Petra Hellwig and Frédéric Melin

\section{Volume 8 Open-Chain Oligopyrrole Systems}

37. BODIPY ${ }^{\circledR}$ Dyes and Their Derivatives: Syntheses and Spectroscopic Properties

Aurore Loudet and Kevin Burgess

38. Supramolecular Chemistry of Pyrrole-Based $\pi$-Conjugated Acyclic Anion Receptors

Hiromitsu Maeda

39. The Synthesis and Properties of Dipyrrins Tabitha E. Wood, Md. Imam Uddin and Alison Thompson

40. Coordination Chemistry of Verdohemes and Open-Chain Oligopyrrole Systems Involved in Heme Oxidation and Porphyrin Destruction Alan L. Balch and Faye L. Bowles

41. Beyond Dipyrrins: Coordination Interactions and Templated Macrocyclizations of Open-Chain Oligopyrroles Martin Bröring

\section{Volume 9 Electronic Absorption Spectra - Phthalocyanines}

42. UV-Visible Absorption Spectroscopic Properties of Phthalocyanines and Related Macrocycles Takamitsu Fukuda and Nagao Kobayashi

\section{Volume 10 Catalysis and Bio-Inspired Systems - Part I}

43. Metalloporphyrin-Catalyzed Asymmetric Atom/Group Transfer Reactions Joshua V. Ruppel, Kimberly B. Fields, Nicole L. Snyder and $X$. Peter Zhang

44. High-Valent Iron-Oxo Porphyrins in Oxygenation Reactions Sam P. de Visser and Wonwoo Nam

45. On the Significance of Phthalocyanines in Solar Cells M. Victoria Martínez-Díaz and Tomás Torres

46. Artificial Photosynthetic Systems Composed of Porphyrins and Phthalocyanines Shunichi Fukuzumi 
47. Anchoring of Porphyrins and Phthalocyanines on Conductors and Semiconductors for Use in Hybrid Electronics Florence Duclairoir and Jean-Claude Marchon

48. Bioinspired Catalysts with $\mathrm{B}_{12}$ Enzyme Functions Yoshio Hisaeda and Hisashi Shimakoshi

\section{Volume 11 Catalysis and Bio-Inspired Systems - Part II}

49. Bio-inspired Molecular Devices Based on Systems Found in Photosynthetic Bacteria

Pierre D. Harvey, Christine Stern and Roger Guilard

50. Self-Assembled Porphyrin Nanostructures Craig J. Medforth and John A. Shelnutt

51. Photochemistry of Chlorophylls and Their Synthetic Analogs Hitoshi Tamiaki and Michio Kunieda

52. Chemistry, Biology and Medical Effects of Water-Soluble Metalloporphyrins

Ines Batinić-Haberle, Júlio S. Rebouças, Ludmil Benov and Ivan Spasojević

\section{Volume 12 Applications}

53. Liquid Crystals of Phthalocyanines, Porphyrins and Related Compounds Kazuchika Ohta, Huy-Danh Nguyen-Tran, Lisa Tauchi, Yoriko Kanai, Takashi Megumi and Yasufumi Takagi

54. Porphyrin-Based Chemical Sensors Roberto Paolesse, Donato Monti, Sara Nardis and Corrado Di Natale

55. Electropolymerized Thin Films of Metalloporphyrins for Electrocatalysis and Electroanalysis

Sophie Griveau and Fethi Bedioui

56. Application of Porphyrins and Related Compounds as Optical Oxygen Sensors

Ichiro Okura and Toshiaki Kamachi

57. Applications of Nanoparticles Containing Porphyrins and Related Systems

Sara Bonacchi, Damiano Genovese, Riccardo Juris, Fabrizio Mancin, Marco Montalti, Luca Prodi, Enrico Rampazzo and Nelsi Zaccheroni 


\section{Volume 13 Synthesis and Structural Studies}

58. The Synthesis of $\pi$-Extended Porphyrins Andrei V. Cheprakov

59. Noncovalent Syntheses of Multiporphyrin Species in Aqueous Solution Rosaria Lauceri, Alessandro D'urso and Roberto Purrello

60. Porphyrins and Porphyrin Analogs Bearing Double Bonds at One or More Meso Positions

Chang-Hee Lee, Vladimir Roznyatovskiy, Seong-Jin Hong and Jonathan L. Sessler

61. The Structural Chemistry of Isolated Chlorophylls Mathias O. Senge and Stuart A. MacGowan

62. Porphyrin-Based Tectons in Molecular Tectonics Véronique Bulach and Mir Wais Hosseini

\section{Volume 14 Theoretical, Electron Transfer and Physical Studies}

63. The Role of Heme-Nitrosyls in the Biosynthesis, Transport, Sensing, and Detoxification of Nitric Oxide (NO) in Biological Systems:

Enzymes and Model Complexes

Nicolai Lehnert, Timothy C. Berto, Mary Grace I. Galinato and Lauren E. Goodrich

64. Chemistry of Sandwich Tetrapyrrole Rare Earth Complexes Yongzhong Bian, Yuexing Zhang, Zhongping Ou and Jianzhuang Jiang

65. Theoretical Aspects of the Optical Spectroscopy of Porphyrinoids Martin J. Stillman

66. Synthesis and Reactivity of High-Valent Transition Metal Corroles and Corrolazines Amanda J. McGown, Yosra M. Badiei, Pannee Leeladee, Katharine A. Prokop, Serena DeBeer and David P. Goldberg

\section{Volume 15 Biochemistry of Tetrapyrroles}

67. Eukaryotic Heme Trafficking Scott Severance, Caiyong Chen and Iqbal Hamza

68. Ferrochelatase Structure and Reaction Mechanism Gloria C. Ferreira and Gregory A. Hunter

69. Heme Proteins as Gas Sensors Emily E. Weinert and Michael A. Marletta 
70. Heme Biosynthesis

Gunhild Layer, Dieter Jahn and Martina Jahn

71. Mechanisms of Cytoprotection by Hemopexin Ann Smith

72. Mechanisms of Heme Uptake and Utilization in Bacterial Pathogens Angela Wilks and Kylie D. Barker

73. Emerging Roles of Heme as a Signal and a Gas-Sensing Site: Heme-Sensing and Gas-Sensing Proteins Jotaro Igarashi, Kenichi Kitanishi and Toru Shimizu

\section{Volume 16 Synthetic Developments - Part I}

74. Carbaporphyrins and Related Systems. Synthesis, Characterization, Reactivity and Insights into Porphyrinoid Aromaticity Timothy D. Lash

75. Synthesis and Properties of the Hybrid PhthalocyanineTetrabenzoporphyrin Macrocycles Andrew N. Cammidge, Isabelle Chambrier, Michael J. Cook and Lydia Sosa-Vargas

\section{Volume 17 Synthetic Developments - Part II}

76. Syntheses of Bacteriochlorins and Isobacteriochlorins Christian Brückner, Lalith Samankumara and Junichi Ogikubo

77. The Hemiporphyrazines and Related Systems Christopher J. Ziegler

78. Electrochemistry of Pyrroles and Oligopyrroles: Analytic and Synthetic Aspects Christophe Bucher and Mihai Buda

\section{Volume 18 Applications and Materials}

79. Ordered Surface Structures of Self-Assembled Porphyrins Nico Veling, Johannes A. A. W. Elemans, Roeland J. M. Nolte and Alan E. Rowan

80. Porphyrin- and Phthalocyanine-Based Solar Cells Hiroshi Imahori, Kei Kurotobi, Michael G. Walter, Alexander B. Rudine and Carl C. Wamser

81. Structures and Properties of Non-Planar Tetrapyrroles Jonathan P. Hill, Shinsuke Ishihara and Katsuhiko Ariga 
82. Porphyrin-Based Nanocomposites for Biosensing

Jianping Lei and Huangxian Ju

83. Electrochemical Organic Electronics Based On Robust Thin-Films of Porphyrazines

Simon Dalgleish and Kunio Awaga

84. Bioconjugation of Phthalocyanine Derivatives

Pui-Chi Lo, Janet T. F. Lau and Dennis K. P. Ng

85. Transition Metal Complexes of Porphyrins and Porphyrinoids Andrey Mironov

\section{Volume 19 Biochemistry of Tetrapyrroles - Part II}

86. Biochemistry of Methyl-CoM Reductase and Coenzyme $\mathrm{F}_{430}$ Yuzhen Zhou, Dariusz Sliwa and Stephen Ragsdale

87. Peroxidase and Heme Thiolate Enzymes Thomas L. Poulos

88. Biosynthesis of Siroheme, Coenzyme $\mathrm{F}_{430}$ and Heme $d_{1}$ Gunhild Layer and Martin J. Warren

89. Diversity of Heme Proteins in Sulfate Reducing Bacteria Célia V. Romão, Margarida Archer, Susana A. Lobo, Ricardo O. Louro, Inês A. C. Pereira, Lígia M. Saraiva, Miguel Teixeira and Pedro M. Matias

90. Dioxygen-Generating Chlorite Dismutases and the CDE Protein Superfamily Jennifer L. DuBois and Jeffery A. Mayfield

91. Side Chain Modification During Heme Biosynthesis John D. Phillips

92. The Cytochrome $b_{561}$ Protein Family Shin-ichi J. Takayama and A.Grant Mauk

93. Heme Attachment to Cytochromes $c$ Julie M. Stevens and Stuart J. Ferguson

\section{Volume 20 Chlorophylls and Related Systems}

94. Key Enzymes of Chlorophyll Biosynthesis Markus J. Broecker, Dieter Jahn and Jürgen Moser

95. Supramolecular Chlorophyll Assemblies for Artificial Photosynthesis Victoria L. Gunderson and Michael R. Wasielewski

96. Bacteriochlorophyll Biosynthesis and Assembly in Green Chlorophototrophic Bacteria: Theme and Variations Zhenfeng Liu and Donald Bryant 
97. Tetrapyrrole Biosynthesis in Plant Systems

Tatsuru Masuda and Koichi Kobayashi

98. Chlorophyll Metabolism in Photosynthetic Organisms

Ryouich Tanaka, Atsushi Takabayashi, Hisashi Ito and Ayumi Tanaka 